\title{
Notch1 is associated with the differentiation of human bone marrow-derived mesenchymal stem cells to cardiomyocytes
}

\author{
ZIPU YU*, YU ZOU*, JINGYA FAN, CHENGCHEN LI and LIANG MA \\ Department of Cardiac Surgery, First Affiliated Hospital, Zhejiang University, Hangzhou, Zhejiang 310000, P.R. China
}

Received October 8, 2015; Accepted August 16, 2016

DOI: $10.3892 / \mathrm{mmr} .2016 .5862$

\begin{abstract}
Notch signaling is involved in the early process of differentiation to determine the fate of stem cells. However, the precise role of Notch in human bone marrow-derived mesenchymal stem cells (hBMSCs) remains unclear. The present study aimed to investigate the involvement of Notch signalling during the course of hBMSC differentiation into cardiomyocytes using hBMSCs, with multilineage differentiation ability, isolated and purified from human bone marrow. Flow cytometric analysis revealed that CD29, CD44 and CD90 were highly expressed on the surface of cells in their fifth passage, whereas detection of CD34, CD45, CD54 and HLA-DR was negative. Visualization of morphological changes, western blotting, immunocytochemistry and reverse transcription-quantitative polymerase chain reaction (RT-qPCR) demonstrated that hBMSCs differentiate into cardiomyocytes through treatment with 5-azacytidine (5-aza). Transmission electron microscopy revealed ultramicroscopic details of differentiated hBMSCs. Western blotting and immunocytochemistry demonstrated increased protein expression levels of $\alpha$-actin and cardiac troponin $\mathrm{T}$ expression, and RT-qPCR revealed increased mRNA expression of Notch1 early in the process of differentiation (days 1, 4 and 7), and increased mRNA expression levels of the transcription factors GATA binding protein- 4 and NK2 homeobox 5 at day 28 day. In conclusion, differentiation of hBMSCs into cardiomyocytes was induced in vitro by 5-aza, and was associated with upregulation of Notch1, GATA binding protein-4 and Nkx2.5 expression. Overexpression of the Notch1 signaling pathway may represent a potential mechanism underlying the differentiation of hBMSCs.
\end{abstract}

Correspondence to: Dr Liang Ma, Department of Cardiac Surgery, First Affiliated Hospital, Zhejiang University, 79 Qinchun Road, Hangzhou, Zhejiang 310000, P.R. China

E-mail:649833863@qq.com

*Contributed equally

Key words: human bone marrow-derived mesenchymal stem cells, differentiation, Notch1, 5-azacytidine

\section{Introduction}

Stem cells are a major focus of modern scientific research into the treatment of heart diseases, as their plasticity may help overcome the limited self-repair capacity of cardiomyocytes. Human bone marrow-derived mesenchymal stem cells (hBMSCs) have been widely used in numerous studies and clinical trials due to their unique properties, including ease of isolation, expansion in vitro, multipotency and immunological tolerance (1-4). Previous studies have revealed that 5-azacytidine (5-aza) induces MSC differentiation into myocardial cells (5-7). It has also been demonstrated that hBMSCs can differentiate into cardiac troponin $\mathrm{T}(\mathrm{cTnT})$-expressing cardiomyocytes (2,8-11); however, the precise mechanisms controlling this process currently remain unclear.

The mechanism of differentiation is complex and requires various signaling pathways. Notch signaling, which is important in cell fate specification during embryogenesis, has previously been implicated in regulating differentiation of stem cells in adults (12). Numerous studies have demonstrated that Notch signaling regulates a wide variety of processes during embryonic and post-natal development, including proliferation, apoptosis and cell fate decisions (13-15). However, the association between Notch signalling and the differentiation of hBMSCs to cardiomyocytes remains unclear. The aim of the present study was to investigate the function of Jagged1-Notch1 signaling during hBMSCs differentiation into cardiomyocytes. As a result of its widespread expression, it was hypothesized that Notch signaling may be involved in the differentiation of hBMSCs.

\section{Materials and methods}

Isolation and culture of hBMSCs. hBMSCs were isolated by bone marrow aspiration from the sternums of 20 male patients (age, 23-48 years) with heart valve diseases awaiting cardiac surgery, but were healthy with regard to the circulatory system. Informed consent was obtained from all patients prior to inclusion in the study. The study used a protocol approved by the Research Ethics Committee of the First Affiliated Hospital, Zhejiang University (Hangzhou, China). Bone marrow mononuclear cells were purified by Ficoll-Paque density-gradient centrifugation as previously described (16). The purified mononuclear cells were allowed to adhere to culture flasks in Dulbecco's modified Eagle's medium (Gibco; Thermo Fisher 
Scientific, Inc., Waltham, MA, USA) supplemented with $10 \%$ fetal bovine serum (Invitrogen; Thermo Fisher Scientific, Inc.) overnight at $37^{\circ} \mathrm{C}$ in $5 \% \mathrm{CO}_{2}$. Cells from passages 3-6 were used for subsequent experiments.

Cell viability assay. hBMSCs ( 5,000 cells/well) were seeded into 96-well plates. Following overnight incubation, the medium was removed and Cell Counting Kit 8 (CCK8; Dojindo Molecular Technologies, Inc., Kumamoto, Japan) solution was added to each well and incubated at $37^{\circ} \mathrm{C}$ for $1 \mathrm{~h}$. The absorbance of the solution was measured spectrophotometrically at $450 \mathrm{~nm}$ with a MRX II absorbance reader (Dynex Technologies, Inc., Chantilly, VA, USA). The growth assay was performed each day for 5 days consecutively.

Analysis of hBMSCs by flow cytometry. Culture-expanded cells (passages 3-6) were washed with phosphate-buffered saline (PBS) containing $0.5 \%(\mathrm{w} / \mathrm{v})$ bovine serum albumin (BSA), their concentration adjusted to $1 \times 10^{6}$ cells $/ 100 \mu \mathrm{l}$, and phenotypic analyses performed via flow cytometry. The hBMSCs were blocked with $1 \%$ BSA and incubated with phycoerythrin- or fluorescein isothiocyanate-conjugated mouse monoclonal antibodies against human CD34 (catalog no. sc-19587; Santa Cruz Biotechnology, Inc., Dallas, TX, USA), CD54 (catalog no. ab27582; Abcam, Cambridge, UK), CD45 (catalog no. 560976; BD Biosciences, San Jose, CA, USA), CD44 (catalog no. 560977; BD Biosciences), CD29 (catalog no. sc-59829; Santa Cruz Biotechnology, Inc.), human leukocyte antigen-antigen D related (HLA-DR; catalog no. 560944; BD Biosciences) and CD90 (catalog no. 561969; BD Biosciences) for $60 \mathrm{~min}$ in the dark at $4^{\circ} \mathrm{C}$, at dilutions recommended by the manufacturers. Subsequently, cells were washed with PBS and fixed with $2 \%$ paraformaldehyde. Immunoglobulin isotype incubation was performed as a negative control. Flow cytometry was performed with a FACSCalibur system (FC500; Beckman Coulter, Inc., Brea, CA, USA) and analysed using FlowJo software version 7.6.5 (FlowJo, LLC, Ashland, OR, USA).

Multilineage differentiation assays. To induce osteogenic differentiation, hBMSCs were cultured in a commercially available osteogenic differentiation medium (catalog no. HUXMA-90021; Cyagen Biotechnology Co. Ltd., Taicang, China). On day 21, cells were stained with Alizarin Red in accordance with the manufacturer's protocol. To induce adipogenic differentiation, hBMSCs were cultured in a commercially available adipogenic differentiation medium (catalog no. HUXMA-90031; Cyagen Biotechnology Co. Ltd.). On day 21, cells were stained for $30 \mathrm{~min}$ with Oil Red O, diluted 3:2 with distilled water and filtered. To induce chondrogenic differentiation, hBMSCs were cultured in a commercially available chondrogenic differentiation medium purchased from Cyagen (catalog no. HUXMA-90041; Cyagen Biotechnology Co. Ltd.). On day 28 , cells were stained with $1 \mathrm{mg} / \mathrm{ml}$ Alcian blue for 30 min. Cells were observed under a Nikon Eclipse E200 light microscope (Nikon Corporation, Tokyo, Japan).

hBMSC5-aza-induced differentiation to cardiomyocytes. Following the third passage, the cells were washed twice with PBS, adjusted to a density of $2 \times 10^{4}$ cells $/ \mathrm{ml}$, and seeded in a 6 -well plate. Following $24 \mathrm{~h}$ incubation, $10 \mu \mathrm{mol} / \mathrm{l} 5$-aza was
Table I. Primers used for quantitative polymerase chain reaction.

\begin{tabular}{ll} 
Gene & \multicolumn{1}{c}{ Primer sequence (5'-3') } \\
\hline GAPDH & F AAGGTGAAGGTCGGAGTCA \\
& R GGAAGATGGTGATGGGATTT \\
Jagged1 & F AGCTATTTGCCGACAAGGCT \\
Notch-1 & F CACTGCCAGGGCTCATTACA \\
& F AACGCCTACCTCTGCTTCTG \\
GATA-4 & R CTCACAGGCACACTCGTAGC \\
NKx2.5 & R GGAAGCCCAAGAACCTGAAT \\
& F TTACCATAGTGAGATGACAG \\
& R GCCAACAACAACTTCGTGAAC
\end{tabular}

GAPDH, glyceraldehyde 3-phosphate dehydrogenase; F, forward; $\mathrm{R}$, reverse; GATA-4, GATA binding protein 4; Nkx2.5, NK2 homeobox 5 .

added (for control group, $0 \mu \mathrm{mol} / 15$-aza was added), and cells were incubated for a further $24 \mathrm{~h}$. The culture medium containing 5-aza was then removed and complete culture medium was added. The cells went through the same treatment process course following each cell passage. The cells were maintained in culture for 4 weeks following treatment. Samples were taken for detection of morphological changes by transmission electron microscopy (TEM) on day 28 , for $\alpha$-actin and cTnT expression by immunocytochemistry and western blot analysis on day 28 , and for analysis of mRNA expression by reverse transcription-quantitative polymerase chain reaction (RT-qPCR) for GATA binding protein-4 (GATA-4), and NK2 homeobox 5 (Nkx2.5) on day 28, and Jagged1 and Notch1 on days 1, 4 and 7.

TEManalysis. Cultured cells were rinsed with PBS and immersed in $2.5 \%$ glutaraldehyde for $4 \mathrm{~h}$, rinsed in $0.1 \mathrm{~mol} / \mathrm{l}$ sodium cacodylate buffer ( $\mathrm{pH} 7.3$ ), and postfixed for $1 \mathrm{~h}$ in $1 \% \mathrm{OsO}_{4}$. The cells were embedded in Epon resin and cut into 60-nm thick sections with a Sorvall MTB2 ultramicrotome (Thermo Fisher Scientific, Inc.). Sections were stained for $20 \mathrm{~min}$ at room temperature with uranyl acetate and lead citrate and examined under a Hitachi H-600 electron microscope (Hitachi, Ltd., Tokyo, Japan).

$R N A$ isolation and RT-qPCR. Total RNA was extracted using TRIzol (Invitrogen; Thermo Fisher Scientific, Inc.) following the manufacturer's protocol and reverse transcribed into cDNA using the PrimeScript RT reagent kit (Takara Biotechnology Co., Ltd., Dalian, China). The resulting cDNA was quantified by qPCR using SYBR Green (Takara Biotechnology Co., Ltd.) and an ABI 7500 fast real-time PCR System (Applied Biosystems; Thermo Fisher Scientific, Inc.). An initial denaturation step was performed at $95^{\circ} \mathrm{C}$ for $30 \mathrm{sec}$, followed by 40 cycles of denaturation at $95^{\circ} \mathrm{C}$ for $30 \mathrm{sec}$ and annealing at $60^{\circ} \mathrm{C}$ for $30 \mathrm{sec}$. Glyceraldehyde 3-phosphate dehydrogenase (GAPDH) mRNA was used as an internal control. The mRNA and miRNA expression levels were normalized to GAPDH mRNA. The quantification cycle $(\mathrm{Cq})$ value of mRNA was calculated using the $2^{-\Delta \Delta \mathrm{Cq}}$ method (17). The qPCR primers 

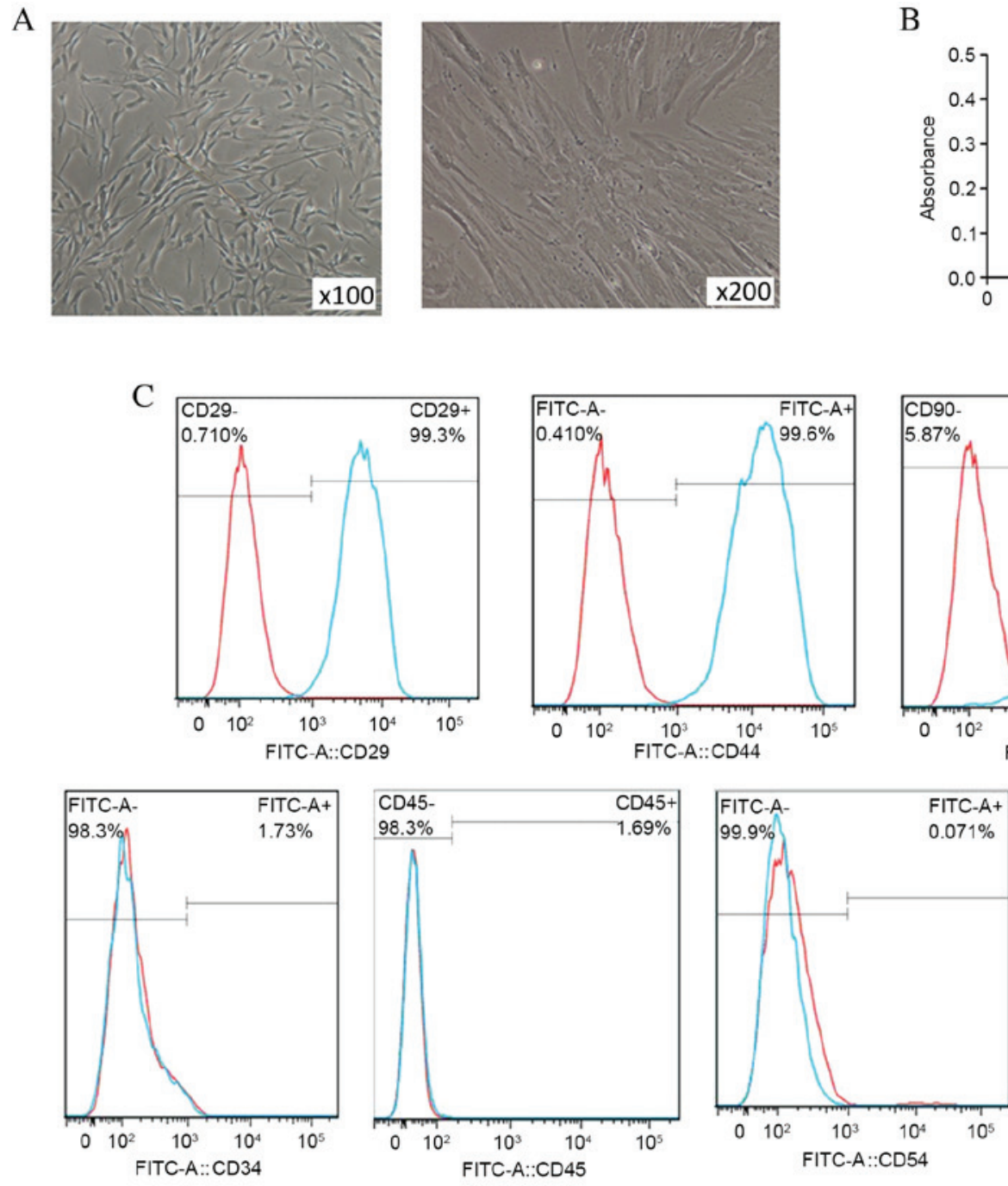

Figure 1. Characterization of hBMSCs. (A) Representative images demonstrating stromal cell-like morphology in hBMSCs. (B) Normalized growth curve demonstrating in vitro expansion ability of hBMSCs. (C) Phenotypic profile of hBMSCs determined by flow cytometry using labeled antibodies specific for the indicated human surface antigens (blue line) and isotype antibodies (red line). hBMSCs, human bone marrow-derived mesenchymal stem cells; FITC, fluorescein isothiocyanate; HLA, human leukocyte antigen.

were provided by Sangon Biotech Co., Ltd. (Shanghai, China) and the sequences are presented in Table I.

Protein extraction and western blotting. The cells were lysed in cell lysis buffer (Sangon Biotech Co., Ltd.). A bicinchoninic acid protein assay kit (Pierce; Thermo Fisher Scientific, Inc.) was used to calculate the total protein concentration in every lysate. Equivalent amounts of protein samples $(100 \mu \mathrm{g})$ were separated on $10 \%$ gels by sodium dodecyl sulphate-polyacrylamide gel electrophoresis and transferred to polyvinylidene difluoride membranes. Membranes were blocked in $10 \%$ skimmed milk for $1 \mathrm{~h}$ at room temperature and then incubated overnight at $4^{\circ} \mathrm{C}$ with the following primary antibodies: Rabbit anti- $\alpha$-actin (catalog no. ab137346; Abcam) and rabbit anti-cTnT (catalog no. ab45932; Abcam), used at dilutions recommended by the manufacturer. Following washing, membranes were incubated with the corresponding goat anti-rabbit secondary antibody (catalog no. D111018; Sangon Biotech Co., Ltd.) for $1 \mathrm{~h}$ at a dilution recommended by the manufacturer. Protein bands were visualized using

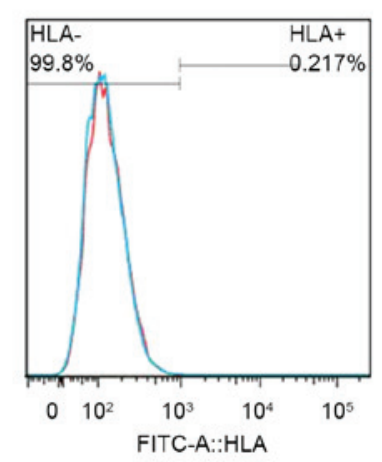

B
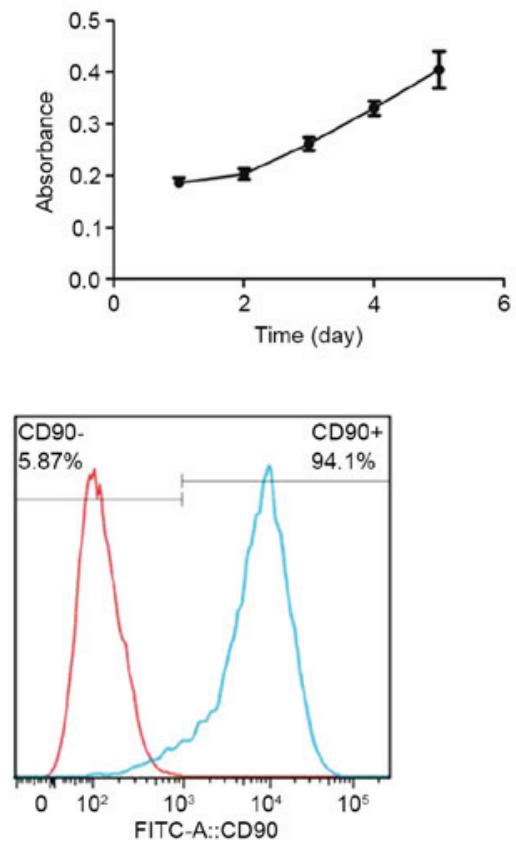

RapidStep $^{\text {TM }}$ Enhanced Chemiluminescence reagent (EMD Millipore, Billerica, MA, USA).

Immunocytochemistry analysis. For immunocytochemical analyses, $1-5 \times 10^{6}$ cells $/ \mathrm{ml}$ were seeded onto glass slides and allowed to adhere overnight. The following day the cells were washed in PBS and fixed for 10-15 min with $3 \% \mathrm{H}_{2} \mathrm{O}_{2}$. Non-specific binding was prevented by blocking with $5 \%$ normal goat serum (Sangon Biotech Co., Ltd.). The cells were then incubated for $2-3 \mathrm{~h}$ at $37^{\circ} \mathrm{C}$ with the $\alpha$-actin and cTnT primary antibodies described previously, washed with PBS, and incubated with the secondary antibody, biotinylated anti-rabbit IgG (catalog no. D111053; Sangon Biotech Co., Ltd.), at a dilution recommended by the manufacturer. Horseradish peroxidase was used as detection reagent and finally diaminobenzidine was used to visualize antibody binding using a Nikon Eclipse E200 light microscope (Nikon Corporation).

Statistical analysis. All data are expressed as the mean \pm standard deviation of three independent experiments. 
Differences between samples were analyzed by $t$-tests using SPSS version 17.0 software (SPSS, Inc., Chicago, IL, USA). $\mathrm{P}<0.05$ was considered to indicate a statistically significant difference.

\section{Results}

Isolation and characterization of hBMSCs in culture. hBMSCs grew as adherent monolayers with a tendency to grow in clusters, and had the morphological appearance of spindle-shaped cells (Fig. 1A). The proliferation ability of the cells was confirmed using CCK-8 (Fig. 1B). Expression of CD105, CD73 and CD90, and lack of expression of CD45, CD34, CD14 or CD11b, CD79a or CD19, and HLA-DR are used as markers to define and detect MSCs. Additionally, MSCs typically differentiate to osteoblasts and adipocytes in vitro (18). Flow cytometry data revealed positive staining for CD29, CD44 and CD90, and negative staining for CD34, CD45, CD54 and HLA-DR, indicating that the isolated hBMSCs were of mesenchymal origin and of high purity (Fig. 1C).

Confirmation of the differentiation capacity of hBMSCs in vitro. The differentiation capacity of hBMSCs into mesodermal lineages (osteocytes, adipocytes and chondrocytes) was assessed in cells cultured in commercially available differentiation media. Alizarin Red staining demonstrated mineralization during osteogenic differentiation in hBMSCs on day 21 (Fig. 2A). Adipogenic differentiation of hBMSCs was characterized by Oil Red O staining, with lipid droplets visible in the differentiated adipocytes on day 21 following the induction of differentiation (Fig. 2B). Positive Alcian blue staining of sections from hBMSC pellets following culture in chondrogenic medium demonstrated the chondrogenic differentiation capabilities of the adherent cells (Fig. 2C).

Morphological changes of hBMSCs in response to treatment with 5-aza. Phase contrast microscopy was used to determine the morphological changes of 5-aza-treated hBMSCs following 7, 14, 21 and 28 days of treatment. Images of untreated cells on days 7, 14, 21 and 28 are presented in Fig. 3A-D. In the experimental group, certain adherent cells died, whereas the surviving cells proliferated and differentiated. Following 7 and 14 days of treatment, cell morphology did not appear to change (Fig. 3E and F, respectively), remaining comparable to the spindle-shaped morphology of undifferentiated cells demonstrated in Fig. 1A. Following 21 and 28 days of treatment, the appearance of spindle-shaped cells was reduced, with cells developing a broadened and flattened shape (Fig. $3 \mathrm{G}$ and $\mathrm{H}$, respectively). TEM on day 28 also revealed a cardiomyocyte-like ultrastructure of sarcomeres, suggesting differentiation of hBMSCs into cardiomyocytes (Fig. 3I). No sarcomeres were observed in undifferentiated cells.

Expression of $\alpha$-actin and cTnT is increased in 5-aza-treated cells. Immunocytochemistry demonstrated that 5 -aza treatment for 28 days induced cTnT and $\alpha$-actin expression in hBMSCs, whereas no cTnT or $\alpha$-actin expression was observed in the untreated control group (Fig. 4A and B, respectively).
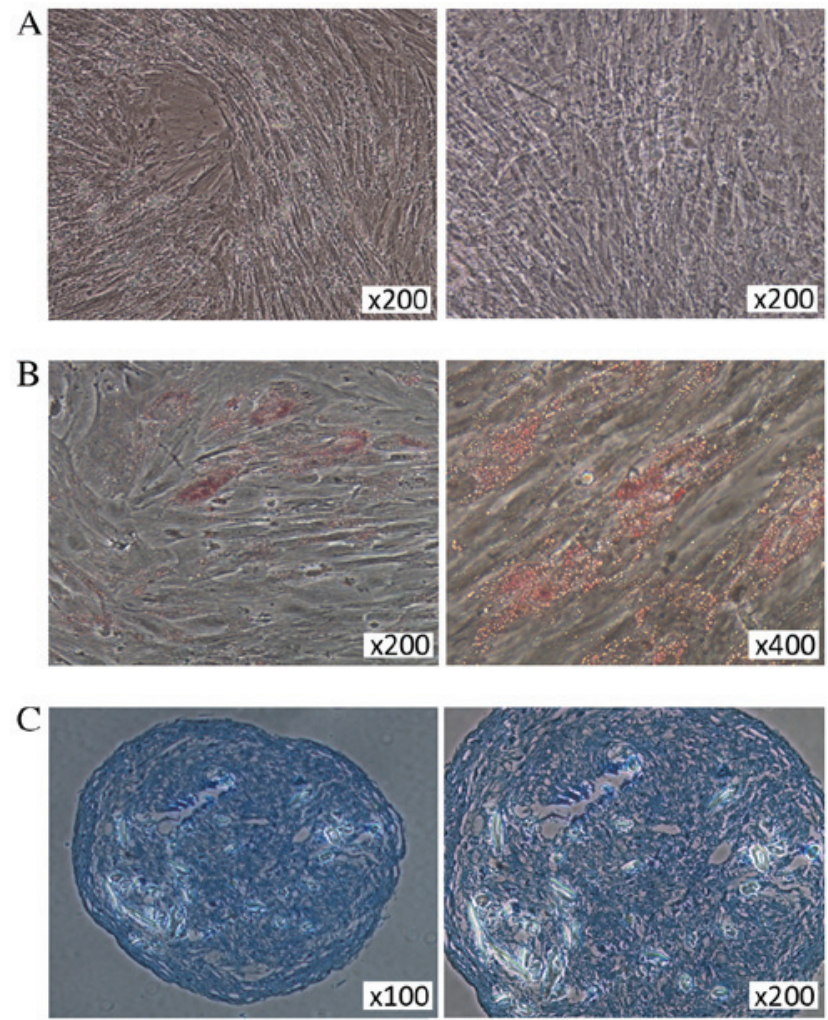

Figure 2. The morphology of hBMSCs and hBMSCs differentiated into osteocytes, adipocytes and chondrocytes using commercially available media. (A) Differentiated osteocytes were stained for mineralization with Alizarin Red on day 21. (B) Differentiated adipocytes were stained for lipid droplets with Oil Red O on day 21. (C) Differentiated chondrocytes were stained for sulphate sugar amino polysaccharide with Alcian blue on day 28. hBMSCs, human bone marrow-derived mesenchymal stem cells

Western blot analysis also demonstrated increased protein expression levels of cTnT and $\alpha$-actin in the 5-aza-treated cells, with no cTnT and $\alpha$-actin detectable in the untreated control group (Fig. 4C and D, respectively). These results indicated that certain hBMSCs in the 5-aza-treated group had undergone differentiation, giving rise to the expression of molecular markers of cardiomyocytes.

RT-PCR analysis for expression of transcription factors and Notch signals in hBMSCs. RT-qPCR analysis indicated that mRNA expression levels of GATA-4 and Nkx2.5 were significantly higher in the 5-aza-treated compared with the untreated control group following 28 days of treatment $(\mathrm{P}=0.012$ and $\mathrm{P}=0.018$, respectively; Fig. $5 \mathrm{~A}$ and $\mathrm{B}$, respectively). During the process of 5-aza-induced hBMSC differentiation, RT-qPCR analysis revealed that Notch1 mRNA expression levels were significantly upregulated compared with untreated control cells on days 1, 4 and 7 of treatment $(\mathrm{P}=0.021, \mathrm{P}=0.016$ and $\mathrm{P}=0.008$, respectively; Fig. 5C). Jagged1 mRNA expression levels in cells treated with 5-aza did not differ significantly compared with untreated control cells on days 1 and 4, but 5-aza treatment did result in significant upregulation on day 7 compared with untreated control cells ( $\mathrm{P}=0.035$; Fig. 5D). These results suggested that the Notch1 signaling pathway and Jagged1 ligand contributed to the differentiation process, during which GATA- 4 and $\mathrm{Nkx} 2.5$ were crucial. 
A
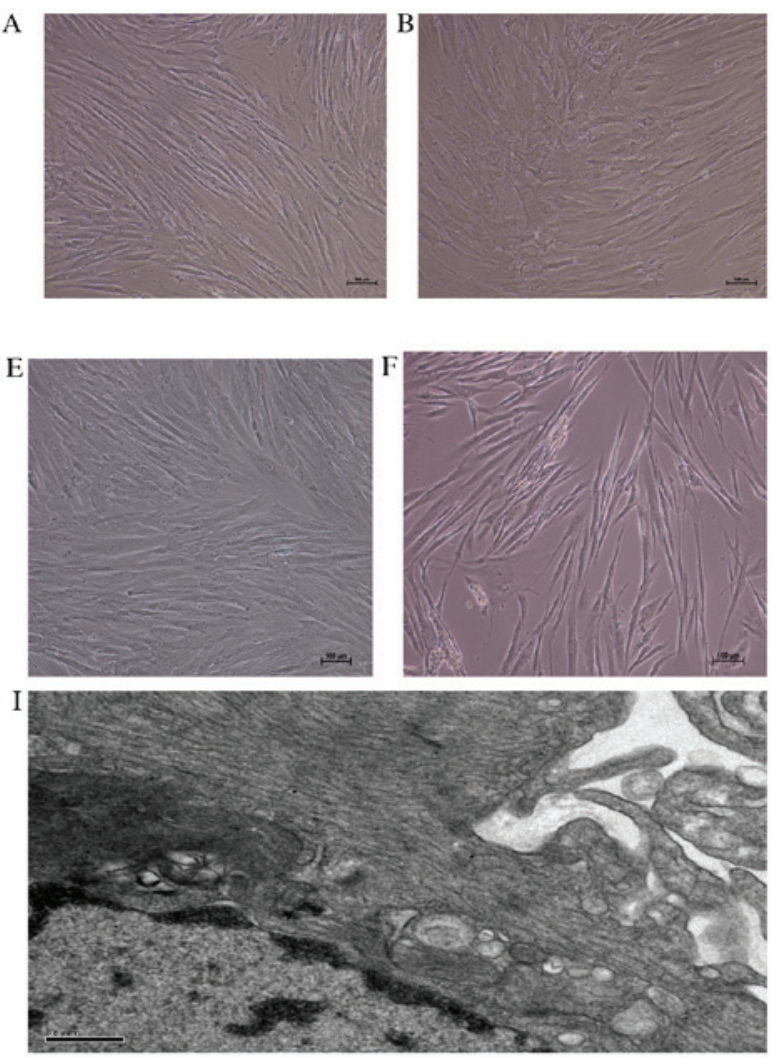
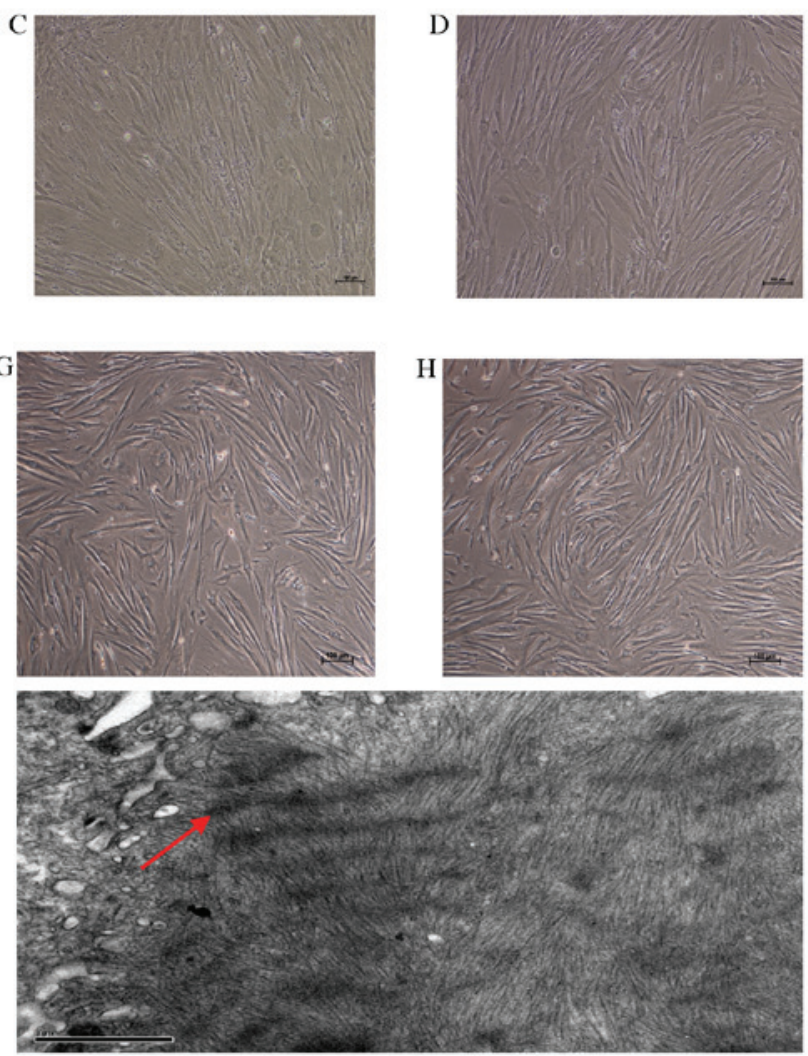

Figure 3. Phenotypic changes of human bone marrow-derived mesenchymal stem cells following treatment with 5-aza. Phase contrast microscopy of untreated cells on (A) day 7, (B) day 14, (C) day 21 and (D) day 28. Phase contrast microscopy on (E) day 7, (F) day 14, (G) day 21 and (H) day 28 of treatment with $10 \mu \mathrm{mol} / 1$ 5-aza. Magnification, x200. (I) Transmission electron microscopy on day 28 of treatment. Sarcomere formation is indicated by the arrow. 5-aza, 5-azacytidine.

\section{Discussion}

MSCs possess characteristics which make them a promising source for cell therapy in numerous diseases (19), with BMSCs currently serving as the primary source of harvested MSCs. The present study revealed that hBMSCs have a doubling time of $\sim 5$ days, which may be due to samples having been obtained from patients awaiting surgery. However, a population of cells, isolated from bone marrow and expanded in culture, is considered to represent an acceptable source of undifferentiated MSCs for investigation of MSC differentiation (20-22). The present study revealed that hBMSCs have adipogenic, osteogenic and chondrogenic differentiation potential in vitro, providing further evidence of the multilineage differentiation potential of hBMSCs that has previously been demonstrated $(23,24)$.

In the present study, hBMSCs exhibited cardiomyocyte characteristics when treated with 5-aza, as evidenced by increased expression of $\alpha$-actin and cTnT by immunocytochemistry and western blotting. The ultramicroscopic details observed by TEM in the 5-aza-treated hBMSCs indicated myocardial differentiation.

In mammals, there are four Notch genes (Notch1-4) and five Notch ligands, Jagged1 and 2, and Delta-like ligands 1, 3 and 4. Upon ligand binding to Notch at the cell membrane, the cytoplasmic domain is cleaved and released from the cell-surface by Presenilin $/ \gamma$-secretase-dependent proteolysis. The cleaved intracellular Notch protein is translocated to the nucleus, where it converts the protein recombination signal binding protein for immunoglobulin $\kappa \mathrm{J}$ region from a transcriptional repressor to a transcriptional activator $(14,25,26)$.

Notch signalling is part of an evolutionarily ancient mechanism of cell interaction, with previous studies having demonstrated Notch signaling at all stages of development, influencing differentiation, proliferation, and apoptotic events (27-29). Notch family proteins have been demonstrated to be expressed in a wide range of mammalian cells and tissues, and are particularly involved in the formation and development of the heart (3,30-32).

The present study also examined mRNA expression levels of important genes of the Notch pathway during the process of differentiation, and demonstrated that mRNA expression levels of Notch1, but not Jagged1, increased significantly on days 1, 4 and 7 of 5-aza treatment, compared with untreated cells. The mRNA expression levels of GATA-4 and Nkx2.5 were also increased significantly on day 28 in the 5-aza-treated cells compared with untreated cells. However, the lack of significant difference in Jagged1 mRNA expression between the 5-aza-treated cells and untreated control cells on days 1 and 4, despite the significant difference on day 7, indicated that Jagged1 may be not the predominant ligand involved in the Notch signalling pathway during the 5-aza-induceddifferentiation of hBMSCs into cardiomyocytes. However, the upregulation of Notch1 mRNA expression levels and the associated transcription factors, GATA-4 and Nkx2.5, suggest that Notch1 signaling 
A

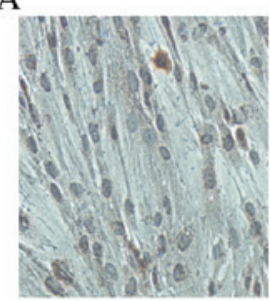

5-aza-treated

C

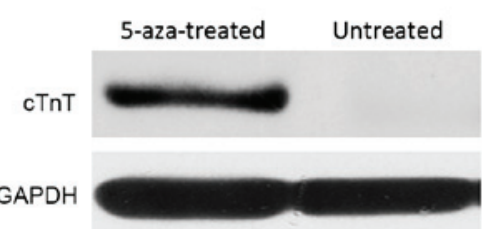

Untreated
B
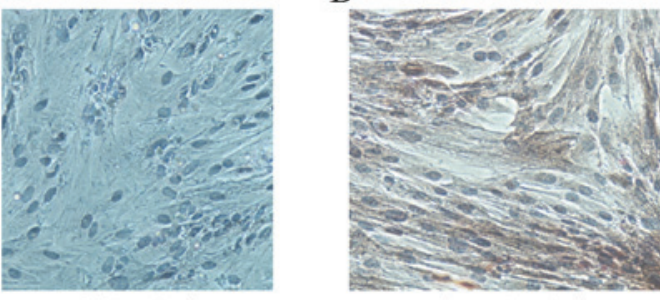

5-aza-treated

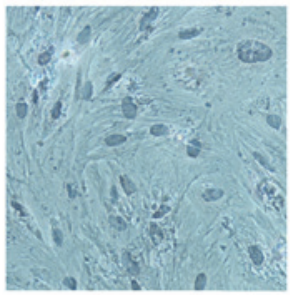

Untreated

D

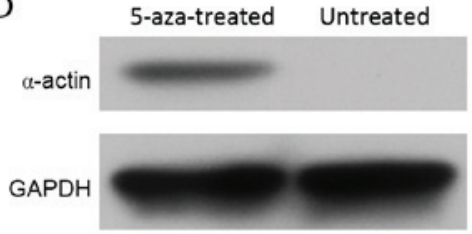

Figure 4. Expression of $\mathrm{cTnT}$ and $\alpha$-actin in hBMSCs following 28 days of 5-aza treatment. Expression of (A) cTNT and (B) $\alpha$-actin in 5-aza-treated and untreated control hBMSCs detected by immunocytochemistry (original magnification, x200). Western blot analysis of protein expression levels of (C) cTnT and (D) $\alpha$-actin alongside GAPDH loading control. hBMSCs, human bone marrow-derived mesenchymal stem cells; 5-aza, 5-azacytidine; cTnT, cardiac troponin T; GAPDH, glyceraldehyde 3-phosphate dehydrogenase.

A

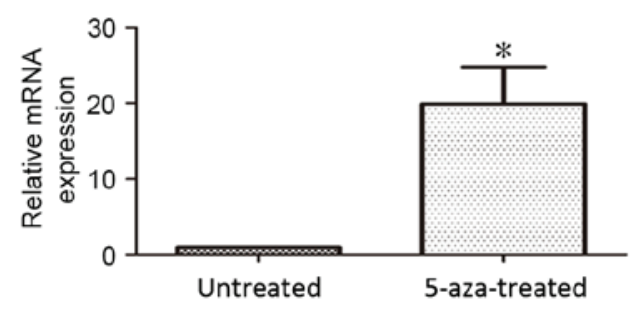

$\mathrm{C}$

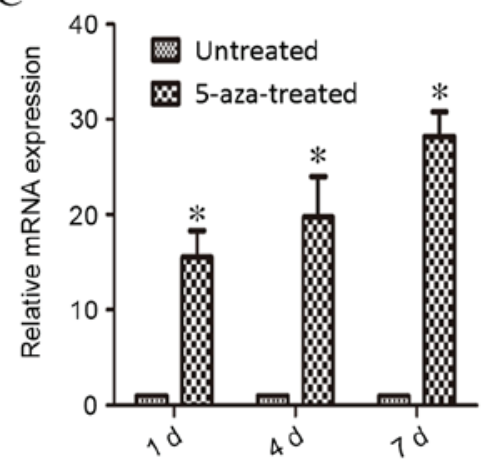

B

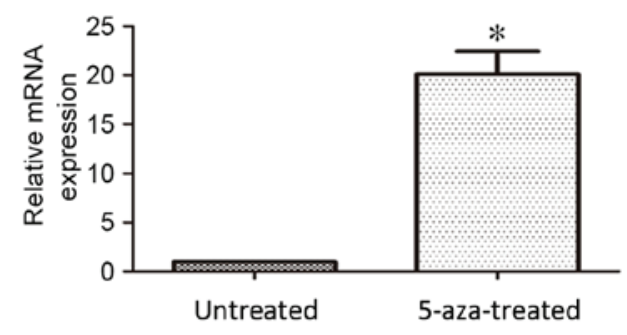

D

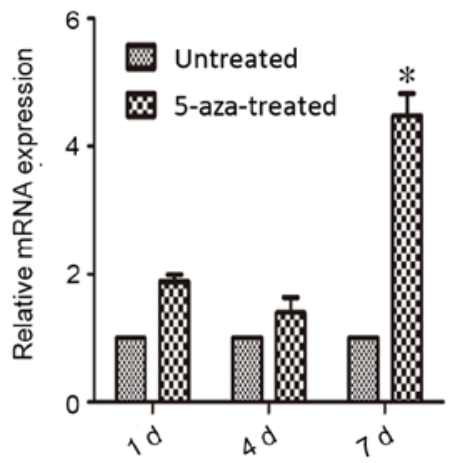

Figure 5. mRNA expression of transcription factors and Notch signals in hBMSCs. Levels of (A) GATA-4, (B) Nkx2.5, (C) Notch1 and (D) Jagged1 were assessed in human bone marrow-derived mesenchymal stem cells following 5-aza treatment by reverse transcription-quantitative polymerase chain reaction. GATA-4 and Nkx2.5 mRNA levels were assessed following 28 days of 5-aza treatment, Notch1 and Jagged1 levels were assessed on days 1, 4 and 7 of treatment. "P<0.05 vs. untreated control. GATA-4, GATA binding protein-4; Nkx2.5, NK2 homeobox 5; 5-aza, 5-azacytidine.

is involved in the differentiation of hBMSCs into cardiomyocytes. Further studies are required to evaluate the degree to whichNotch1 promotes this process.

Certain previous studies have demonstrated that the expression of $\mathrm{Nkx} 2.5$ and GATA-4, both of which are involved in the development of the heart (33), is enhanced during 5-aza-induced differentiation of hBMSCs into myocardial cells. This is consistent with the results of the present study.
In conclusion, the results of the present study demonstrated that Notch1, GATA-4 and Nkx2.5 are upregulated during the differentiation process of hBMSCs induced by 5 -aza. hBMSC differentiation may be partially regulated through the transcription factors, GATA-4 and Nkx2.5. As an important signaling pathway, Notch1 may exert a regulatory effect on the differentiation process. By identifying the functions of Notch1, it may be possible to influence the Notch1 signaling pathway to regulate hBMSC differentiation induced by 5 -aza. 


\section{Acknowledgements}

We thank all members of State Key Laboratory for Diagnosis and Treatment of Infectious Diseases for providing us with laboratory apparatus and technical support. This work was supported by National Natural Science Funding of China (grant no. 81070264).

\section{References}

1. Kopen GC, Prockop DJ and Phinney DG: Marrow stromal cells migrate throughout forebrain, and cerebellum and they differentiate into astrocytes after injection into neonatal mouse brains. Proc Natl Acad Sci USA 96: 10711-10716, 1999.

2. Pittenger MF, Mackay AM, Beck SC, Jaiswal RK, Douglas R, Mosca JD, Moorman MA, Simonetti DW, Craig S and Marshak DR: Multilineage potential of adult human mesenchymal stem cells. Science 284: 143-147, 1999.

3. Saito T, Dennis JE, Lennon DP, Young RG and Caplan AI: Myogenic Expression of mesenchymal stem cells within myotubes of mdx mice in vitro and in vivo. Tissue Eng 1: 327-343, 1995.

4. Williams AR and Hare JM: Mesenchymal stem cells: Biology, pathophysiology, translational findings, and therapeutic implications for cardiac disease. Circ Res 109: 923-940, 2011.

5. Yang S, Huang S, Feng C and Fu X: Umbilical cord-derived mesenchymal stem cells: Strategies, challenges, and potential for cutaneous regeneration. Front Med 6: 41-47, 2012.

6. Wu CX, Zhang EY, Yang SY, Ma JY, An Q and Shi YK: The expression of GATA-4 and Nkx2.5 gene in the transformation of Rattus mesenchymal stem cells into cardiomyocytes. Sichuan Da Xue Xue Bao Yi Xue Ban 39: 882-885, 2008 (In Chinese).

7. Cho J, Rameshwar P and Sadoshima J: Distinct roles of glycogen synthase kinase (GSK)-3alpha and GSK-3beta in mediating cardiomyocyte differentiation in murine bone marrow-derived mesenchymal stem cells. J Biol Chem 284: 36647-36658, 2009.

8. Rangappa S, Entwistle JW, Wechsler AS and Kresh JY: Cardiomyocyte-mediated contact programs human mesenchymal stem cells to express cardiogenic phenotype. J Thorac Cardiovasc Surg 126: 124-132, 2003 .

9. Liechty KW, MacKenzie TC, Shaaban AF, Radu A, Moseley AM, Deans R, Marshak DR and Flake AW: Human mesenchymal stem cells engraft and demonstrate site-specific differentiation after in utero transplantation in sheep. Nat Med 6: 1282-1286, 2000.

10. Orlic D, Kajstura J, Chimenti S, Limana F, Jakoniuk I, Quaini F, Nadal-Ginard B, Bodine DM, Leri A and Anversa P: Mobilized bone marrow cells repair the infarcted heart, improving function and survival. Proc Natl Acad Sci USA 98: 10344-10349, 2001.

11. Kudo M, Wang Y, Wani MA, Xu M, Ayub A and Ashraf M: Implantation of bone marrow stem cells reduces the infarction and fibrosis in ischemic mouse heart. J Mol Cell Cardiol 35 1113-1119, 2003.

12. Yuan TM and Yu HM: Notch signaling: Key role in intrauterine infection/inflammation, embryonic development, and white matter damage? J Neurosci Res 88: 461-468, 2010.

13. Reddy BV, Rauskolb C and Irvine KD: Influence of fat-hippo and notch signaling on the proliferation and differentiation of Drosophila optic neuroepithelia. Development 137: 2397-2408, 2010.

14. Weinmaster G: The ins and outs of notch signaling. Mol Cell Neurosci 9: 91-102, 1997.
15. Gridley T: Notch signaling in vertebrate development and disease. Mol Cell Neurosci 9: 103-108, 1997.

16. Li J, Tao R, Wu W, Cao H, Xin J, Li J, Guo J, Jiang L, Gao C, Demetriou AA, et al: 3D PLGA scaffolds improve differentiation and function of bone marrow mesenchymal stem cell-derived hepatocytes. Stem Cells Dev 19: 1427-1436, 2010.

17. Livak KJ and Schmittgen TD: Analysis of relative gene expression data using real-time quantitative PCR and the 2(-Delta Delta C(T)) method. Methods 25: 402-408, 2001.

18. Horwitz EM, Le Blanc K, Dominici M, Mueller I, Slaper-Cortenbach I, Marini FC, Deans RJ, Krause DS and Keating A; International Society for Cellular Therapy: Clarification of the nomenclature for MSC: The international society for cellular therapy position statement. Cytotherapy 7: 393-395, 2005.

19. Si YL, Zhao YL, Hao HJ, Fu XB and Han WD: MSCs: Biological characteristics, clinical applications and their outstanding concerns. Ageing Res Rev 10: 93-103, 2011.

20. Tosh D and Slack JM: How cells change their phenotype. Nat Rev Mol Cell Biol 3: 187-194, 2002.

21. Badorff C, Brandes RP, Popp R, Rupp S, Urbich C, Aicher A, Fleming I, Busse R, Zeiher AM and Dimmeler S: Transdifferentiation of blood-derived human adult endothelial progenitor cells into functionally active cardiomyocytes. Circulation 107: 1024-1032, 2003.

22. Spees JL, Olson SD, Ylostalo J, Lynch PJ, Smith J, Perry A, Peister A, Wang MY and Prockop DJ: Differentiation, cell fusion, and nuclear fusion during ex vivo repair of epithelium by human adult stem cells from bone marrow stroma. Proc Natl Acad Sci USA 100: 2397-2402, 2003.

23. Hou M, Yang KM, Zhang H, Zhu WQ, Duan FJ, Wang $H$, Song YH, Wei YJ and Hu SS: Transplantation of mesenchymal stem cells from human bone marrow improves damaged heart function in rats. Int J Cardiol 115: 220-228, 2007.

24. Schilling T, Nöth U, Klein-Hitpass L, Jakob F and Schütze N: Plasticity in adipogenesis and osteogenesis of human mesenchymal stem cells. Mol Cell Endocrinol 271: 1-17, 2007.

25. Ward EJ, Shcherbata HR, Reynolds SH, Fischer KA, Hatfield SD and Ruohola-Baker H: Stem cells signal to the niche through the Notch pathway in the Drosophila ovary. Curr Biol 16: 2352-2358, 2006.

26. Weinmaster G: Notch signal transduction: A real rip and more. Curr Opin Genet Dev 10: 363-369, 2000.

27. Brenner M: To be or notch to be. Nat Med 6: 1210-1211, 2000.

28. Artavanis-Tsakonas S, Rand MD and Lake RJ: Notch signaling: Cell fate control and signal integration in development. Science 284: 770-776, 1999 .

29. Kojika S and Griffin JD: Notch receptors and hematopoiesis. Exp Hematol 29: 1041-1052, 2001.

30. Artavanis-Tsakonas S, Matsuno K and Fortini ME: Notch signaling. Science 268: 225-232, 1995.

31. Li L, Milner LA, Deng Y, Iwata M, Banta A, Graf L, Marcovina S, Friedman C, Trask BJ, Hood L and Torok-Storb B: The human homolog of rat Jagged1 expressed by marrow stroma inhibits differentiation of 32D cells through interaction with Notch1. Immunity 8: 43-55, 1998.

32. Perdigoto $C N$ and Bardin AJ: Sending the right signal: Notch and stem cells. Biochim Biophys Acta 1830: 2307-2322, 2013.

33. Armiñán A, Gandía C, Bartual M, García-Verdugo JM, Lledó E, Mirabet V, Llop M, Barea J, Montero JA and Sepúlveda P. Cardiac differentiation is driven by NKX2.5 and GATA4 nuclear translocation in tissue-specific mesenchymal stem cells. Stem Cells Dev 18: 907-918, 2009. 